\title{
GRETA - Gamma Ray Energy Tracking Array
}

\author{
I-Yang Lee
}

Nuclear Science Division, Lawrence Berkeley National Laboratory, Berkeley CA 94720

\begin{abstract}
Gamma ray energy tracking is a new concept for detecting gamma rays. It can be implemented as a $4 \pi$ array of highly segmented Ge detector, which would advance detection sensitivity by three orders of magnitude in certain applications. Such an array will provide tremendous discovery potential at current stable and exotic beam facilities, as well as at future facilities. The development of a tracking array for nuclear structure physics has brought together scientists from different areas. Technical advances made in the last 5 years have demonstrated that the construction of a gamma ray energy-tracking array is feasible, and a plan for constructing a US array is in place
\end{abstract}

\section{INTRODUCTION}

Gamma-ray spectroscopy is one of the most powerful tools for the study of a wide range of nuclear properties. Since the beginning of nuclear physics, new discoveries and a deeper understanding of nuclear phenomena have come from major advances in gamma ray detector technology. The most recent step was the introduction of the large $4 \pi$ arrays of Compton-suppressed Ge detectors, Gammasphere and Euroball. They have made a tremendous impact on the scientific productivity and vitality of low energy nuclear physics. This can be seen, for example, from the large number of participants and new results presented here in this conference. These arrays have higher efficiency and provided a 100 -fold increased in the sensitivity over the previous generation of arrays. Previous advances in gamma ray detectors include the improvement of energy resolution by using Ge detector to replace NaI detectors, the increase of efficiency and peak-to-total ratio through the availability of large volume Ge crystals, and the further improvement of the efficiency and peak-to-total ratio from the construction of Compton suppressed arrays. The recent development of segmented Ge detectors will enable us to further improve the overall efficiency, Compton suppression, position resolution, directional information, and polarization measurements. I will discuss the new concept of a $4 \pi$ gamma ray energy tracking array, which is capable of determining the position and energy deposition of every gamma-ray interaction in the detector and giving another factor of 100 to 1000 gain in sensitivity compared to current arrays for a board range of nuclear physics studies.

Gamma ray energy tracking was first proposed by LBNL in 1994. It takes advantage of the recent advances in detector segmentation, digital electronics, and computing power. At present, the proof-of-principle has been achieved through the substantial R\&D efforts carried out in US, Europe, and Japan. One design of such an array, a US effort called GRETA, is a $4 \pi$ spherical shell of closely packed highly segmented coaxial Ge crystals [1] (plans for a similar array, called AGATA, are being developed in Europe). Using the largest available crystal $(8 \mathrm{~cm}$ diameter) and leaving sufficient internal space for auxiliary detectors (30 $\mathrm{cm}$ diameter), we can construct this array with approximately 100 crystals each with 36 segments. The detailed pulse shapes from each of the segments are obtained by digitizing them at a time step of about $10 \mathrm{nsec}$, and they are used to determine the energy and threedimensional positions of all gamma-ray interactions. This information is used together with the characteristics of Compton scattering and pair production processes to track the scattering of all the gamma rays. Such an array will provide high efficiency, excellent peak-to-total ratio, and accurate position information. 
TABLE 1. GRETA performance.

\begin{tabular}{lcc}
\hline Properties & Gammasphere & GRETA/AGATA \\
\hline Solid angle coverage & 0.45 & 0.8 \\
Efficiency $(1.3 \mathrm{MeV})$ & 0.08 & 0.50 \\
Efficiency $(15 \mathrm{MeV})$ & 0.005 & 0.23 \\
Position resolution & $20 \mathrm{~mm}$ & $2 \mathrm{~mm}$ \\
Peak-to-total ratio $(1.3 \mathrm{MeV})$ & 0.66 & 0.78 \\
Energy resolution $(1.3 \mathrm{MeV})$ & $2.3 \mathrm{keV}$ & $2.0 \mathrm{keV}$ \\
Time resolution $(1.3 \mathrm{MeV})$ & $8 \mathrm{nsec}$ & $5 \mathrm{nsec}$ \\
Direction information & $\mathrm{No}$ & $\mathrm{Yes}$ \\
Polarization sensitivity $(1.3 \mathrm{MeV})$ & 0.04 & 0.3 \\
Counting rate (per detector) & $10 \mathrm{kHz}$ & $50 \mathrm{kHz}$ \\
\hline
\end{tabular}

\section{PERFORMANCE AND PHYSICS OPPORTUNITIES}

A comparison of the expected performance of a gamma ray energy-tracking array with a current generation array, Gammasphere, is shown in Table I. Gammasphere uses Compton (BGO) shields to reject gamma rays that scatter from a crystal and do not deposit their full energy. GRETA can achieve a much higher efficiency due to its $4 \pi$ coverage with Ge detectors and its ability to track, which allows the full energy of "escaped" gamma rays to be recovered by proper summing of interactions in the neighboring detectors (and segments). For example, GRETA has an efficiency which is about 6 times greater than Gammasphere for a $1.3 \mathrm{MeV}$ gamma ray and about 40 times greater for a $15 \mathrm{MeV}$ gamma ray, because at this higher energy the size of the interaction distribution (the shower) is comparable to the size of the crystal. Another important advantage of tracking is that the first interaction can be identified and its position determined with an accuracy of about $2 \mathrm{~mm}$, compared with $20 \mathrm{~mm}$ for Gammasphere. This is critical for Doppler shift correction, especially for experiments where the gamma ray is emitted from nuclei traveling at very high velocities. Other benefits of segmentation, digital signal processing, and tracking are better energy and time resolution, higher polarization sensitivity, and higher counting rate capability.

TABLE 2. Resolving power of tracking detector array. The calculation assumes a solid angle coverage of $80 \%$ and a position resolution of $2 \mathrm{~mm}$.

\begin{tabular}{|l|l|l|l|l|c|}
\hline Reaction & $\begin{array}{l}<\mathbf{E} \gamma> \\
(\mathbf{M e V})\end{array}$ & $\mathbf{v} / \mathbf{c}$ & $\mathbf{M} \gamma$ & $\begin{array}{l}\text { Resolving } \\
\text { Power }\end{array}$ & $\times$ Gammasphere \\
\hline Stopped nuclei & 5.0 & 0.0 & 4 & $2.1 \times 10^{7}$ & 200 \\
\hline High-spin normal kinematics & 1.0 & 0.04 & 20 & $2.4 \times 10^{6}$ & 55 \\
\hline High-spin inverse kinematics & 1.0 & 0.07 & 20 & $2.2 \times 10^{6}$ & 120 \\
\hline Coulex/transfer & 1.5 & 0.1 & 15 & $3.7 \times 10^{6}$ & 510 \\
\hline Fragmentation in-beam & 1.5 & 0.5 & 6 & $5.9 \times 10^{6}$ & 12500 \\
\hline Fragmentation Coulex & 5.0 & 0.5 & 2 & $2.7 \times 10^{3}$ & 110 \\
\hline
\end{tabular}

These unprecedented capabilities will provide new physics opportunities and will meet the challenges of future accelerator facilities. As shown in Table 2, GRETA will have a resolving power, the ability to identify weak 
cascades among a large background, 100 to 1000 times higher than Gammasphere. This will extend the cross section limit of typical in-beam $\gamma$-ray studies from micro barns to nano barns. The improved sensitivity will push the frontiers of nuclear physics to higher spin, higher excitation energy, further from the line of stability, and to heavier elements. For example, the high granularity and high efficiency will allow us to investigate new exotic phenomena (such as hyperdeformed shapes with a 3:1 axis ratio), to study the shell structure of very heavy elements and their stability, and to map out the order-to-chaos transition at high temperature. The much higher efficiency for high energy gamma rays, combined with good energy resolution, will open up new areas of giant resonance (GR) studies. This will allow coincidence experiments of gamma decays of GR with low lying states, including the selection and study of the GR built on states with exotic shapes such as superdeformation. Dramatic changes in the GR energy are predicted in nuclei near the neutron drip line. These nuclei are produced with small cross sections and require the high efficiency of GRETA. The better position resolution will improve the Doppler correction and improve the energy resolution for nuclei decaying at high velocities, such as those produced by inverse kinematics and fragmentation reactions. Detailed wave function measurements of exotic nuclei can be obtained by nucleon knockout reactions, which require gamma-ray coincidences with high-energy resolution to resolve the population of discrete states. In nuclear astrophysics, many important processes of star evolution and explosion involve low energy capture reactions with very small cross sections. These measurements require high detection efficiency, and tracking can further reduce the background by providing information on the direction of the gamma-ray source. The latter capability has also been identified as a method to greatly improve the sensitivity of certain neutrino-less double beta decay experiments and there are plans to use highly segmented coaxial germanium detector in the next generation of such experiments.

In the current US long-range plan of nuclear physics, a Rare Isotope Accelerator (RIA) is the highest priority for major new construction [2,3]. RIA will offer exciting new scientific opportunities, but will also post major challenges in instrumentation. GRETA could meet the challenges of the gamma ray detector. The most exotic RIA beams will be very weak and the high efficiency of GRETA would extend the range of study to more neutron-rich and proton-rich nuclei. The high background from the decay of the radioactive beam can be better handled by GRETA's high rate capability. Most importantly, the RIA beams produced from fragmentation reactions could have a velocity up to $0.7 \mathrm{c}$. The position resolution of GRETA is crucial to recover the good resolution of a Ge detector by accurately correcting the Doppler shift.

\section{HISTORY AND ORGANIZATION}

The concept of tracking and the opportunities of a gamma-ray tracking detector were first discussed in the previous (1996) US long-range plan of nuclear physics. Soon after that the US low energy nuclear physics community began to organize the gamma ray tracking development efforts. An advisory committee was formed in 1999, and in 2001 it was reorganized as a national steering committee. The steering committee has overseen the development efforts, and organized several workshops involving the community to define the physics requirements, the performance goals, and the research and development needs. One recent workshop, on the digital electronics, was held at Argonne (March, 2001) and another general meeting was held at University of Mass at Lowell (June, 2001). Working groups on physics, electronics, detector design, software and auxiliary detectors were organized following these workshops. As a result of these efforts and in particular the successful R\&D work that demonstrated the proof of principle (see below), a $4 \pi$ gamma-ray tracking facility was listed as an important new initiative in the 2002 long range plan. Recently, a gamma ray tacking coordination committee (GRTCC) was convened to coordinate the development of various tracking detector technologies in nuclear structure within the US. In its report, this committee has made a number of recommendations, the main one being that the only viable implementation for a $4 \pi$ tracking array is a shell of closely packed coaxial Ge detectors.

\section{R\&D STATUS}

A tracking array requires a number of new technologies. R\&D efforts carried out successfully in the last five years have shown that building a $4 \pi$ tracking detector array is now feasible. The four main areas where we have demonstrated proof-of-principle are: the production of segmented coaxial Ge detectors: signal digitization for 
detailed shape analysis: position determination of the interactions from the pulse shape: tracking the scattering sequence based on the energy and position of the interactions.

A prototype detector for GRETA with a tapered hexagonal shape and 36 segments was received in 1999. With new low noise preamps [4] the total integrated noise of the detector is $<5 \mathrm{keV}$ at a bandwidth of $35 \mathrm{MHz}$. It gives an excellent energy resolution for all the segments; about $1.9 \mathrm{keV}$ for $1.33 \mathrm{MeV}$ gamma rays and $1.2 \mathrm{keV}$ for $60 \mathrm{keV}$ gamma rays [5]. Extensive position sensitivity measurements have been carried out on this prototype and they demonstrated that the position of a single interaction within a segment could be determined with an accuracy of about $1 \mathrm{~mm}$ in all three dimensions [6]. In these measurements, to ensure a single interaction at a well-defined position, we used a collimated ${ }^{137} \mathrm{Cs}$ source and collimated $\mathrm{NaI}$ detectors to detect the Compton scattered gamma ray at 90 degrees in coincidence with the signal in the Ge detector. The pulse shapes were digitized using commercially available digitizers. The measured pulse shapes, both net signal and induced signals, were compared with calculated signals and good agreement was found.

After we verified the calculated signals, they were used as a reference to determine the position of the interaction points from the measured signals. A number of algorithms have been developed with the goal of achieving both a good position accuracy and fast computational speed. The best position resolution achieved is about $1 \mathrm{~mm}$ and the minimum distance where two interaction points can be separated is about $2 \mathrm{~mm}$.

A $1 \mathrm{MeV}$ gamma ray typically makes three Compton interactions and one photoelectric interaction before it is absorbed in Ge. Knowing the position and energy of the interactions, and using the angle-energy relation of the Compton scattering process, we can determine the correct sequence of scattering (from the $4 !=24$ possible sequences) [7]. By summing only the energy of the interactions belonging to an identified gamma ray we avoid the false summing problem seen in non-segmented detectors under high gamma multiplicity conditions. The position of the first interaction provides the direction of the gamma ray for Doppler correction. Furthermore, tracking can also reject escaped Compton events and gamma rays not coming from the target direction. The pair-production events were identified by their characteristic large energy deposition at one interaction and interactions of two $0.511 \mathrm{MeV}$ gamma rays [8]. Current tracking algorithms can achieve an efficiency of about $50 \%$ at a gamma ray multiplicity of 25, for both Compton and pair production events.
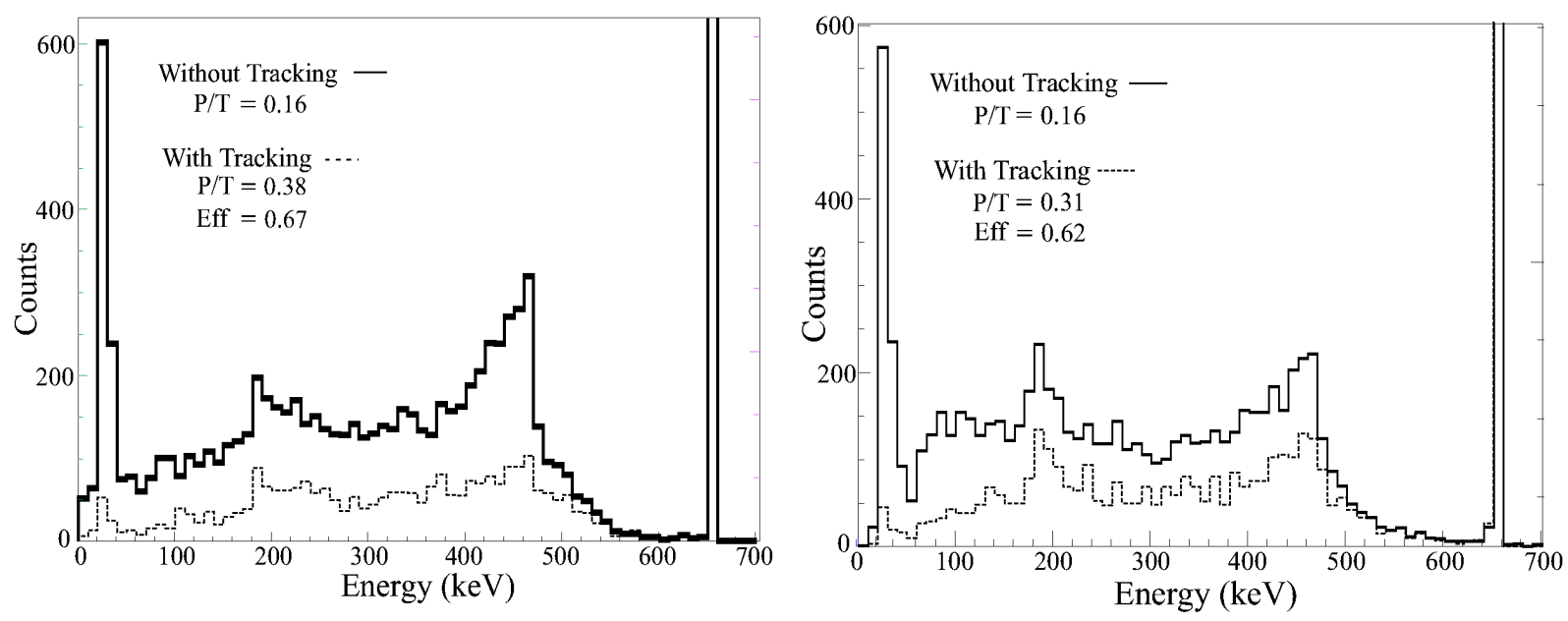

FIGURE 1. Left panel shows the simulated raw spectrum without tracking of $0.662 \mathrm{MeV}$ gamma rays and a spectrum with tracking. The right panel shows the same spectra from actual measurements. The efficiency is relative to the efficiency without tracking.

Recently, we carried out additional tests of the prototype using radioactive sources of ${ }^{60} \mathrm{Co}$, ${ }^{137} \mathrm{Cs}$ and ${ }^{152} \mathrm{Eu}$, as well as an in-beam test using the ${ }^{129} \mathrm{Xe}+{ }^{12} \mathrm{C}$ reaction. In these measurements, most of the segments were instrumented with digital electronics and the full analysis procedure of signal decomposition and tracking was applied to the data. The simulated data were treated with exactly the same analysis procedure as the measurement. Figure 2 compares the measured results with simulated data of the $0.662 \mathrm{MeV}$ gamma ray from ${ }^{137} \mathrm{Cs}$. An algorithm based on the least square method was used for the signal analysis, which can handle up to 4 segments each with 2 
interactions. The efficiency of this method of locating a single interaction in one segment to within $1 \mathrm{~mm}$ is $85 \%$. The measurement and simulation agree with each other for both the raw spectra and the spectra after the tracking. As expected for a single crystal, the tracking improves the peak-to-total ratio but does not increase the efficiency. These results indicate that we have an accurate understanding of the signals from the prototype and all the necessary data analysis algorithms are functioning.

\section{FUTURE PLANS}

Future research and development plans include, additional measurements with the prototype, obtaining a threecrystal detector module, improvement of both signal analysis and tracking algorithms, and development of digital electronics. Our next step in detector prototyping is a three-crystal detector module. Like the previous prototype, the crystals will be $9 \mathrm{~cm}$ long with a tapered regular hexagon shape and 36 segments, but the diameter of the crystals before they are shaped will be $8 \mathrm{~cm}$ rather than $7 \mathrm{~cm}$. As shown in Figure 3, they will be closely packed in one cryostat with a gap of less than $3.5 \mathrm{~mm}$ between the crystals and $4.5 \mathrm{~mm}$ between the crystals and the cryostat walls. We expected to receive this prototype in one year.
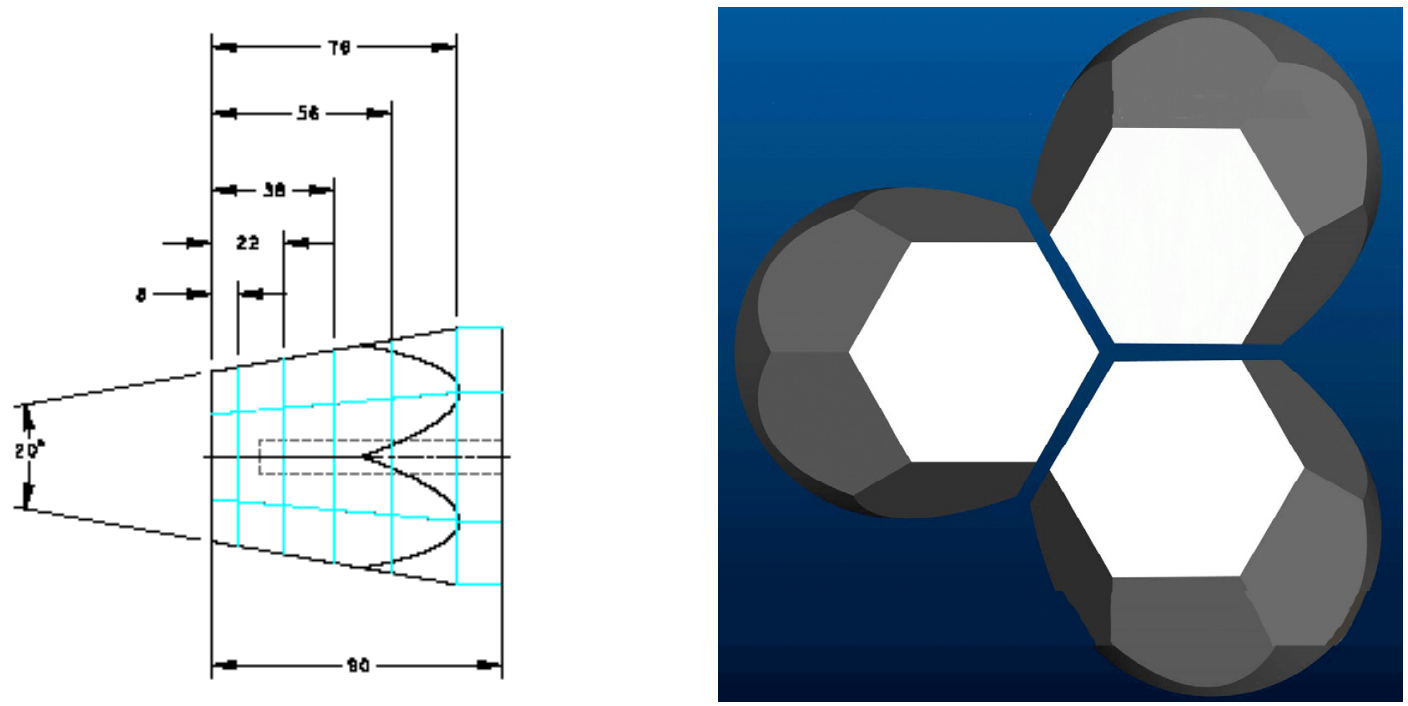

FIGURE 2. Left panel shows shape and segmentation of the crystal in the prototype three crystal detector module (side view). The right panel shows the packing of the crystals in the cryostat (view from the front).

An electronics module for signal digitizing is being developed, based on the specifications determined at a workshop held at ANL. The first version will have eight input channels and provide energy, time, and pulse shape information. Each channel can generate and accept an independent trigger, and the data are read out via a VME bus. The ADC's have a sampling rate of $100 \mathrm{MHz}$ and a resolution of 12 bits. This module will be available for testing at the end of 2002. Based on the experience gained from this module, we will design a 40-channel module capable of processing all the signals and provide positions and energies of all interactions in one crystal.

Currently, we envision that the construction of GRETA can be completed in eight years, and prior to this experiments will be carried out during an 'early implementation' phase.

\section{ACKNOWLEDGMENTS}

The GRETA Steering Committee coordinates the community input and participation of the GRETA development. It is promoting the physics and planning the development effort. Kai Vetter, Greg Schmid, and Austin Kuhn have done most of the development work at LBNL. Dino Bazzacco and Th. Kroell collaborated with the 
LBNL group on the development of signal analysis algorithms. T. Teranishi and N. Aoi collaborated on the development of the algorithm for tracking pair-production events. Rod Clark, Marie-Agnes Deleplanque, Mario Cromaz, Paul Fallon, Augusto Macchiavelli, Frank Stephens, and David Ward have contributed to the effort at LBNL.

\section{REFERENCES}

1. Deleplanque, M. A., et al., Nucl. Instrum. Methods Phys. Res. A430, 292 (1999).

2. Sherrill, B., contribution to this Conference.

3. Savard, G., contribution to this Conference.

4. Yaver, H., private communication.

5. Vetter, K., et al., Nucl. Instrum. Methods Phys. Res. A452, 105 (2000).

6. Vetter, K., et al., Nucl. Instrum. Methods Phys. Res. A452, 223 (2000).

7. Schmid, G.J., et al., Nucl. Instrum. Methods Phys. Res. A430, 69 (1999).

8. Teranishi, T., et al., LBNL NSD 1999 Annual report. 